\title{
The cytosolic glyoxalases of Plasmodium falciparum are dispensable during asexual blood-stage development
}

\author{
Cletus A. Wezena ${ }^{1}$, Romy Alisch ${ }^{1}$, Alexandra Golzmann ${ }^{2}$, Linda Liedgens ${ }^{1}$, Verena Staudacher ${ }^{1,3}$, Gabriele \\ Pradel $^{2}$ and Marcel Deponte ${ }^{1,3, *}$ \\ ${ }^{1}$ Department of Parasitology, Ruprecht-Karls University, D-69120 Heidelberg, Germany. \\ ${ }^{2}$ Division of Cellular and Applied Infection Biology, Institute of Zoology, RWTH Aachen University, D-52074 Aachen, Germany. \\ ${ }^{3}$ Department of Chemistry/Biochemistry, University of Kaiserslautern, D-67663 Kaiserslautern, Germany. \\ * Corresponding Author: \\ Marcel Deponte, Department of Chemistry/Biochemistry, University of Kaiserslautern, Erwin-Schrödinger-Straße 54, D-67663 \\ Kaiserslautern, Germany; E-mail: deponte@chemie.uni-kl.de
}

\begin{abstract}
The enzymes glyoxalase 1 and 2 (Glo1 and Glo2) are found in most eukaryotes and catalyze the glutathione-dependent conversion of 2-oxoaldehydes to 2-hydroxycarboxylic acids. Four glyoxalases are encoded in the genome of the malaria parasite Plasmodium falciparum, the cytosolic enzymes PfGlo1 and PfcGlo2, the apicoplast enzyme PftGlo2, and an inactive Glo1-like protein that also carries an apicoplast-targeting sequence. Inhibition or knockout of the Plasmodium glyoxalases was hypothesized to lead to an accumulation of 2-oxoaldehydes and advanced glycation end-products (AGE) in the host-parasite unit and to result in parasite death. Here, we generated clonal P. falciparum strain 3D7 knockout lines for PFGLO1 and PFCGLO2 using the CRISPR-Cas9 system. Although 3D7Dglo1 knockout clones had an in-

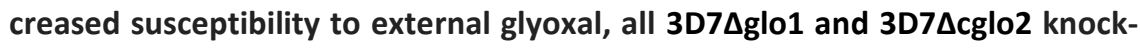
out lines were viable and showed no significant growth phenotype under standard growth conditions. Furthermore, the lack of PfcGlo2, but not PfGlo1, increased gametocyte commitment in the knockout lines. In summary, PfGlo1 and PfcGlo2 are dispensable during asexual blood-stage development while the loss of PfcGlo2 may induce the formation of transmissible gametocytes. These combined data show that PfGlo1 and PfcGlo2 are most likely not suited as targets for selective drug development.
\end{abstract}

doi: $10.15698 /$ mic2018.01.608 Received originally: 04.10.2017; in revised form: 06.11.2017, Accepted 08.11.2017, Published 20.11.2017.

Keywords: Plasmodium falciparum, malaria, glyoxalase, drug target, CRISPR/Cas9.
Abbreviations:
AGE - Advanced glycation end- products,
Glo1 - glyoxalase 1,
Glo2 - glyoxalase 2,
GSH - reduced glutathione,
Pf - Plasmodium falciparum.

\section{INTRODUCTION}

In 1990, Vander Jagt et al. reported that Plasmodium falciparum-infected erythrocytes do not only consume much more glucose than uninfected erythrocytes, but also produce up to 30 -times more D-lactate owing to a highly efficient glyoxalase system [1]. Since this seminal study, the $P$. falciparum glyoxalases have gained considerable attention as model enzymes [2-5] and as potential targets for rational drug development [6-10]. The glyoxalase system, which usually consists of glutathione (or trypanothione in kinetoplastida), the isomerase glyoxalase 1 (Glo1) and the thioesterase glyoxalase 2 (Glo2), is found in most eukaryotes and several prokaryotes [5, 11-13]. The system is considered to be relevant for the detoxification of (glycolysis-derived) methylglyoxal and other 2-oxoaldehydes, thus preventing the formation of advanced glycation end-products (AGE) with implications for diabetes, cancer and many other important diseases [14-19]. However, 2-oxoaldehydes, glyoxalases and AGE can also have regulatory roles, and insular glyoxalases point to alternative functions $[5,13,18,20-25]$.

The $P$. falciparum genome encodes four glyoxalases, two cytosolic and two apicoplast proteins [4, 8, 13]. Cytosolic PfGlo1 and PfcGlo2 presumably constitute a functional glyoxalase system $[4,7,8]$, whereas the Glo1-like protein PfGILP is inactive in standard enzyme assays and cannot provide canonical substrates for apicoplast PftGlo2 because of an altered active site $[4,5,7,8]$. In addition, human erythrocytes harbour a functional hGlo1/hGlo2 couple $[8,26,27]$. We previously characterized the effects of nonglutathione as well as glutathione-derived inhibitors on the two different active sites of recombinant PfGlo1 $[4,9]$. Two 
A

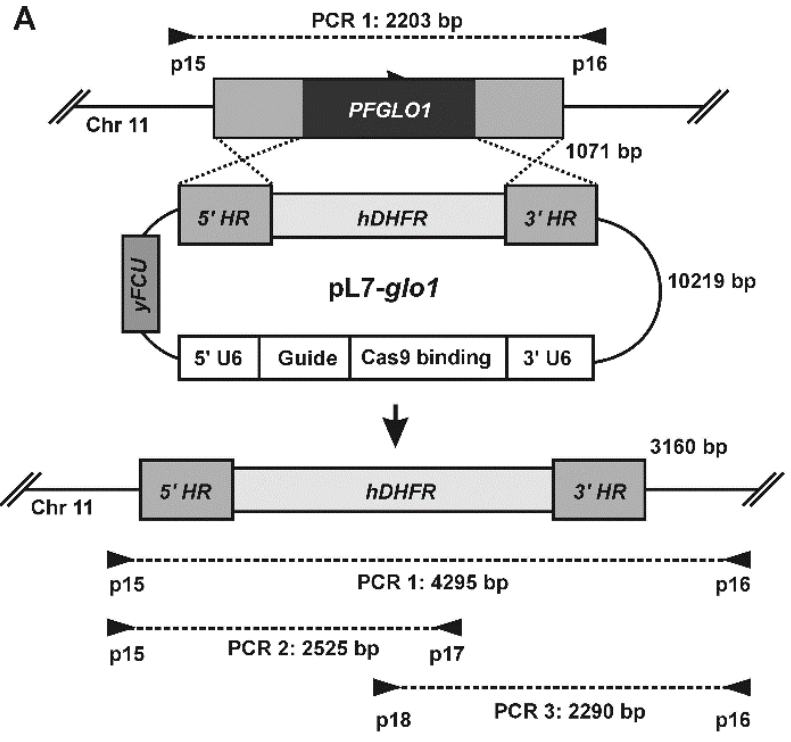

B

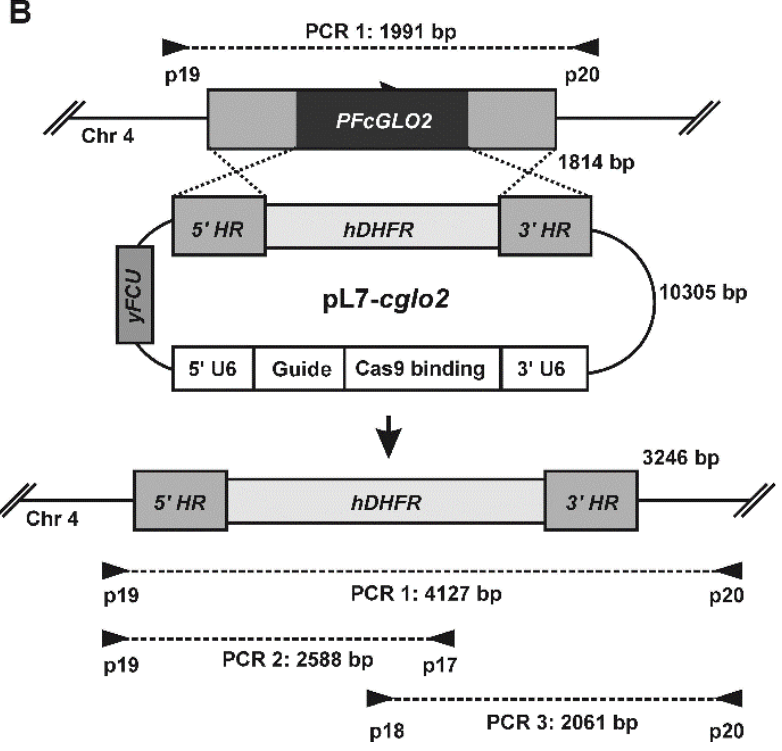

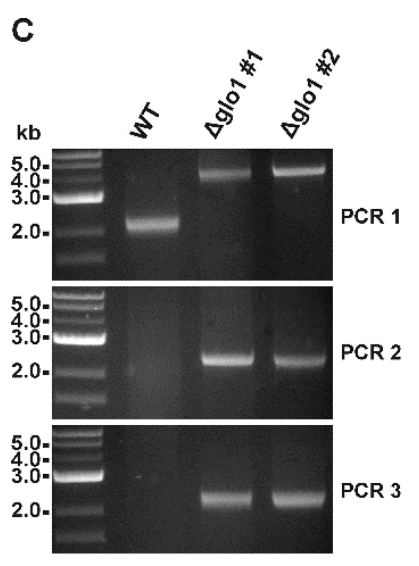

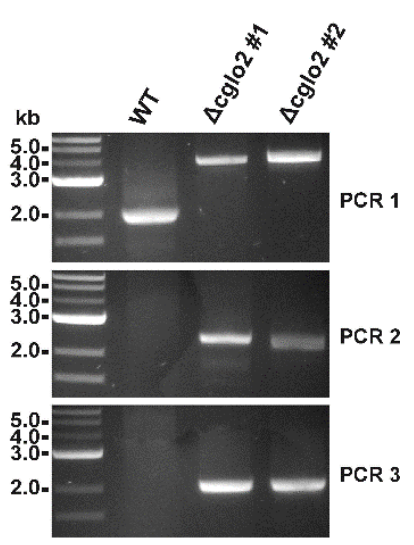

D
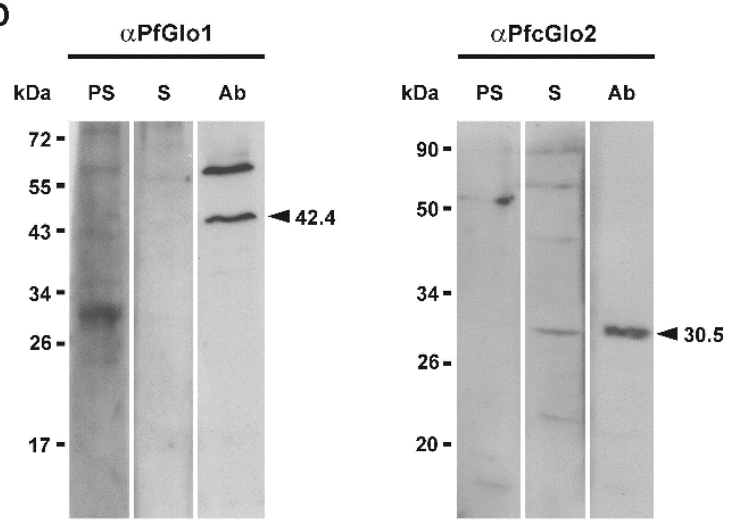

E

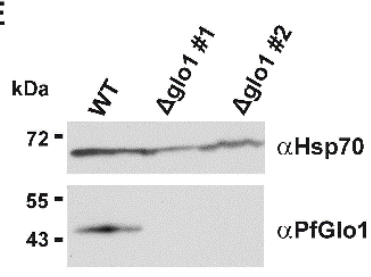

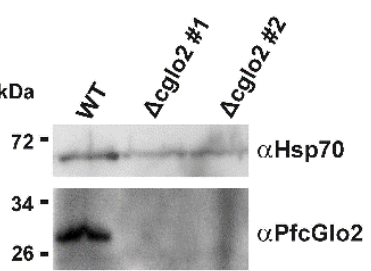

FIGURE 1: Generation and validation of $P$. falciparum 3D7 $\Delta$ glo1 and 3D7Dcglo2 knockout strains. (A) and (B) Knockout strategy for PFGLO1 and PFCGLO2 using the CRISPR-Cas9 system based on the method by Ghorbal et al. [29]. Primer pairs and expected PCR products 1-3 for wild-type strain 3D7 and knockout parasite lines are indicated. (C) PCR controls with isolated genomic DNA from clonal parasite lines 3D7 $\Delta$ glo1 and 3D7 $\Delta$ cglo2 after transfection, selection and limited dilution. PCR products 1-3 are shown for two clones (\#1 and \#2) from each knockout experiment. Wild-type strain 3D7 served as a control. (D) Analysis of rabbit peptide antibodies against PfGlo1 and PfcGlo2. P. falciparum extracts from $2 \times 10^{7}$ cells were loaded per lane on a 15\% gel, separated by reducing SDS-PAGE and analyzed by western blotting. The expected sizes of PfGlo1 and PfcGlo2 are indicated by arrowheads. PS, S, Ab: Decoration with pre-immune serum, serum and affinity-purified antibody, respectively. (E) Western blot controls with extracts from wild-type strain 3D7 as well as two clonal 3D7 $\Delta$ glo1 and 3D7Dcglo2 parasite lines from panel $\mathrm{C}$ using the purified antibodies from panel D. Decoration with an antibody against $\mathrm{Hsp} 70$ served as a loading control.

tight-binding Glo1 inhibitors and a variety of ester derivates were also characterized in cell culture experiments revealing micromolar $\mathrm{IC}_{50}$ values that were three to four orders of magnitude higher than the $\mathrm{IC}_{50}$ values with recombinant PfGlo1 $[9,10]$. Whether the glyoxalase system of the erythrocyte- $P$. falciparum host-parasite unit is indeed a suitable drug target remained to be shown. We therefore analyzed in the present study the relevance of the cytosolic $P$. falciparum glyoxalases for parasite survival using a combination of reverse genetics and biochemical assays. We found that PfGlo1 and PfcGlo2 are both dispensable for asexual blood-stage development while the loss of PfcGlo2 results in increased gametocyte commitment rates. Thus, PfGlo1 and PfcGlo2 are most likely not suited as targets for selective drug development and inhibition of PfcGlo2 might even promote the spread of malaria.

\section{RESULTS}

Generation and validation of glyoxalase knockout strains Initial attempts to disrupt PFGLO1 and PFCGLO2 by double 


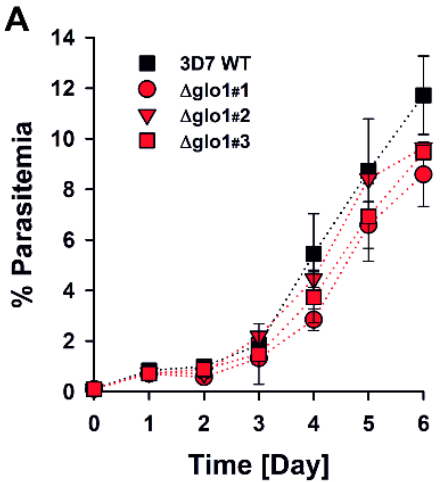

FIGURE 2: Growth curve analysis of $P$. falciparum

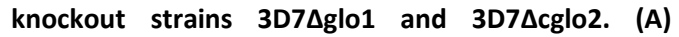
Growth curves of three clonal 3D7 $\Delta$ glo1 parasite lines in

comparison to wild-type strain 3D7. (B) Growth curves of two clonal 3D7 $\Delta$ cglo2 parasite lines in comparison with wild-type strain 3D7. All strains were diluted to an initial parasitemia of $0.1 \%$ and monitored by counting

parasites in Giemsa-stained blood smears. All data points are the mean \pm S.D. from three independent experiments. Statistical analysis using the one way ANOVA method in SigmaPlot 12.5 did not reveal a significant difference among strains $(p>0.05)$. crossover recombination using the plasmid pHTK [28] were not successful despite a variety of selection protocols that combined positive selection with the dihydrofolate reductase inhibitor WR99210 and negative selection with the Herpes simplex thymidine kinase substrate ganciclovir (data not shown). We therefore speculated that both genes might be either essential or that the loci might be inaccessible to genetic manipulation [13]. Following the establishment of the CRISPR-Cas9 system in P. falciparum, we were able to delete both genes using the method by Ghorbal et al. [29] (Fig. 1A, B). Successful deletion by double crossover recombination was validated for clonal

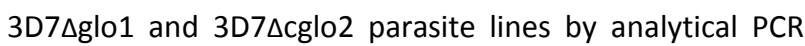
(Fig. 1C). In addition, gene multiplications or other genetic events that might have resulted in functional PfGlo1 or PfcGlo2 were excluded by western blot analysis with specific antibodies (Fig. 1D), revealing that all clonal knockout strains lacked either PfGlo1 or PfcGlo2 as expected (Fig. 1E). In summary, PFGLO1 and PFCGLO2 are not essential for the asexual blood-stages of $P$. falciparum.

\section{Phenotypic analysis and enzyme activities of glyoxalase knockout strains}

The phenotype of clonal 3D7 $\Delta$ glo1 and 3D7 $\Delta$ cglo2 parasite lines was analyzed for Giemsa-stained blood smears by light microscopy. None of the knockout strains had a suspicious morphology during asexual blood-stage development when compared to the wild-type line (Fig. S1). Even though growth rates appeared to be slightly reduced for single knockout clones, differences to the wild-type strain were not significant according to statistical analyses (Fig. 2).

The contribution of PfGlo1 and PfcGlo2 to the overall Glo1 and Glo2 activities in purified parasite extracts was determined by comparing the 3D7 wild-type strain with clonal 3D7 $\Delta$ glo1 and 3D7 $\Delta$ cglo2 parasite lines (Fig. 3). The Glo1 activity of 3D7 $\Delta$ glo1 parasites dropped by approximately $90 \%$ (Fig. 3A), whereas 3D7 $\Delta$ glo2 parasites maintained about one third of the Glo2 activity (Fig. 3B). The latter result might be explained by the presence of (potentially dual localized) PftGlo2 in parasite extracts or the presence of human Glo2 that is taken up into the parasite. A residual Glo1 activity might either originate from a noncanonical parasite enzyme or from human Glo1 that is taken up. It is also worth mentioning that the Glo1 activity might be up-regulated in $3 \mathrm{D} 7 \Delta \mathrm{cgl}$ o2 parasites, even though averaged differences between strain 3D7 and the clonal knockout lines were not significant according to statistical analyses (Fig. 3A).

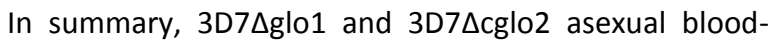
stage parasites have an inconspicuous morphology and no pronounced growth defect. The absent phenotype might be due to residual Glo1 and Glo2 activities that are detectable in purified knockout parasites. While residual Glo2 activities around $30-40 \%$ are plausible in the presence of
A

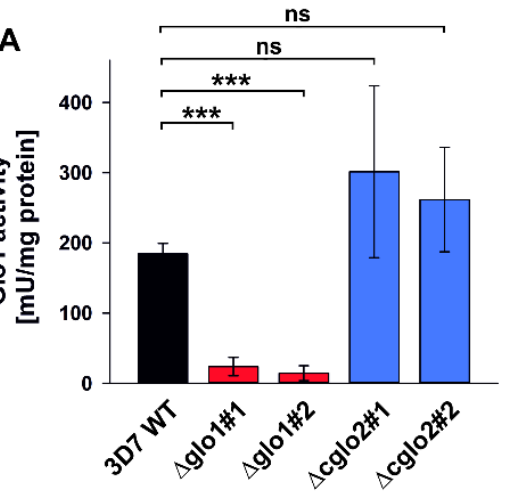

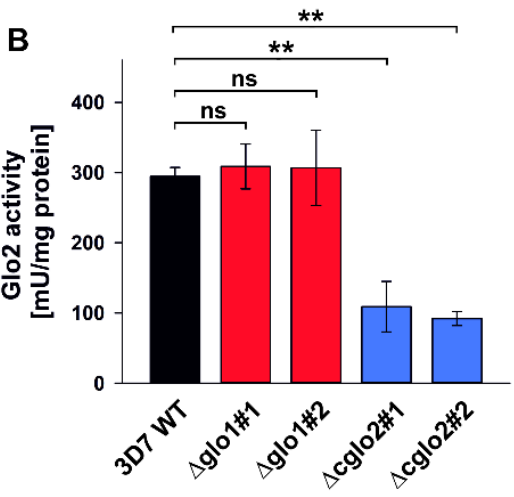

FIGURE 3: Glyoxalase activity measurements of $P$. falciparum knockout

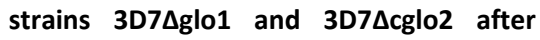
erythrocyte removal by saponin lysis. (A) Normalized Glo1 activity measurements at $240 \mathrm{~nm}$ with parasite extracts from approximately $10^{7} \quad$ trophozoites. (B) Normalized Glo2 activity measurements at $240 \mathrm{~nm}$ with parasite extracts from approximately $1.7 \times 10^{7}$ trophozoites. All data points are the mean \pm S.D. from two to four independent triplicate measurements. Statistical analyses were performed in SigmaPlot 12.5 using the one way ANOVA method (ns, not significant; ** $\mathrm{p}<0.01 ; * * * \mathrm{p}<0.001)$. 
A

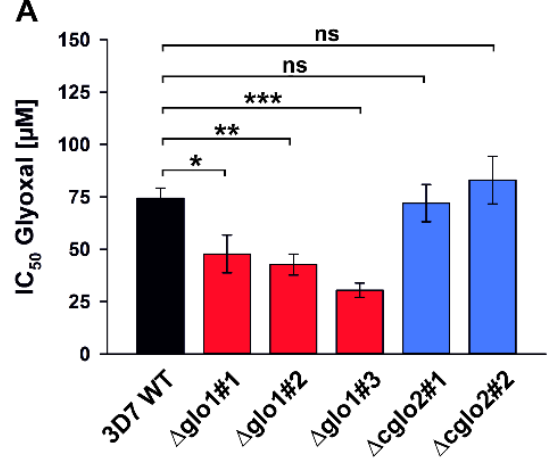

B

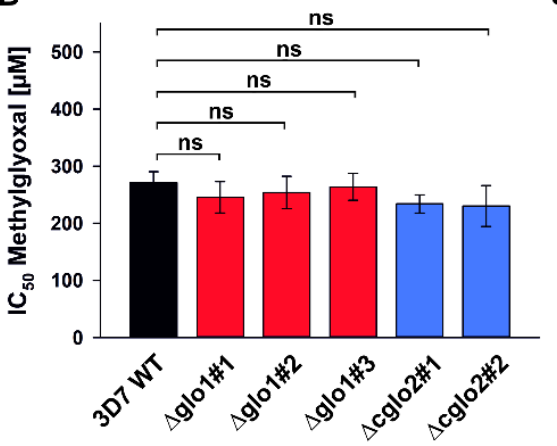

C

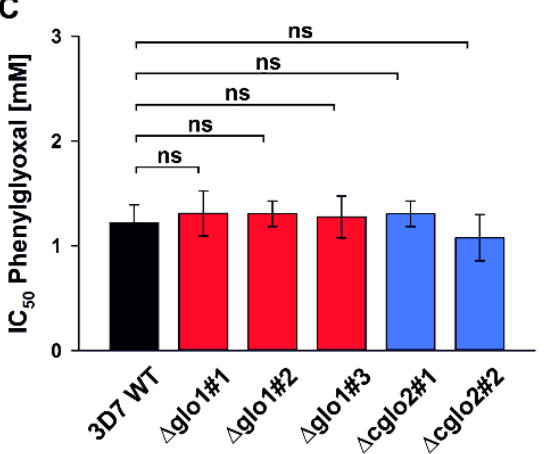

FIGURE 4: Growth inhibitory effects of exogenous 2-oxoaldehydes on synchronous ring-stage cell cultures of $P$. falciparum 3D7 wild-type

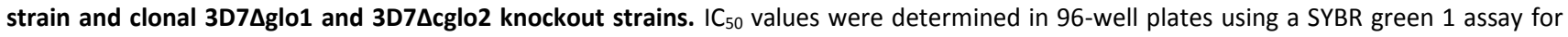
bolus treatments with (A) glyoxal, (B) methylglyoxal and (C) phenylglyoxal after $72 \mathrm{~h}$ incubation. The dose response curves are shown in Fig. S2. All data points are the mean \pm S.D. from technical triplicate measurements of three to seven independent experiments. Statistical analyses were performed in SigmaPlot 12.5 using the one way ANOVA method (ns, not significant; ${ }^{*} p<0.05 ;{ }^{* *} p<0.01 ;{ }^{* * *} p<0.001$ ).

PftGlo2, a residual Glo1 activity around $10 \%$ is quite surprising for 3D7 $\mathrm{g}$ lo1 knockout parasites considering the absence of another functional canonical Glo1-isoform in $P$. falciparum.

\section{Susceptibility of glyoxalase knockout strains to 2-oxoaldehydes}

Next, we analyzed the susceptibility of clonal 3D7 $\Delta$ glo1 and 3D7 $\Delta$ cglo2 parasite lines to external glyoxal, methylglyoxal and phenylglyoxal. Bolus treatments of ring-stage parasites revealed that the toxicity of the 2-oxoaldehydes depended on the size and/or polarity of the compounds with lowest $\mathrm{IC}_{50}$ values for glyoxal and highest IC $\mathrm{I}_{50}$ values for phenylglyoxal (Fig. 4 and Fig. S2). The $I_{50}$ value for glyoxal decreased by up to $60 \%$ for the $3 \mathrm{D} 7 \Delta$ glo1 knockout parasites (Fig. $4 A$ ), whereas no significant differences between wild-type and 3D7 $\Delta$ glo1 knockout parasites were observed for methylglyoxal and phenylglyoxal (Fig. 4B, C). The susceptibility of the clonal 3D7 $\Delta$ cglo2 knockout parasites towards external 2-oxoaldehydes was similar to wild-type parasites (Fig. 4 and Fig. S2). In summary, 3D7 $\Delta$ glo1 knockout parasites have an increased susceptibility towards external glyoxal, which is more toxic for $P$. falciparum than methylglyoxal and phenylglyoxal.

\section{AGE accumulation in glyoxalase knockout strains}

In order to address a potential AGE accumulation in glyoxalase knockout strains, we performed western blot analyses with purified trophozoite-stage parasite extracts using a commercial polyclonal anti-AGE antibody. Staining patterns with this antibody revealed a few bands and smears around 40, 70 and $180 \mathrm{kDa}$ (Fig. 5). A general, unspecific accumulation of modified proteins was not detected. The staining patterns at 70 and $180 \mathrm{kDa}$ were

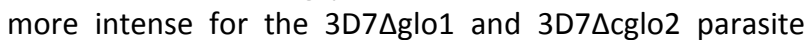
extracts than for wild-type parasite extracts. Band patterns were similar under steady-state conditions in the absence of external 2-oxoaldehydes (Fig. 5A) and $6 \mathrm{~h}$ after a bolus treatment with $50 \mu \mathrm{M}$ glyoxal (Fig. 5B). In summary, western blot analyses support the accumulation of selected modified proteins in glyoxalase knockout strains. However, the growth of asexual blood-stage parasites appears to be unaffected by these protein modifications.

\section{Gametocytogenesis of glyoxalase knockout strains}

In a final set of experiments, we aimed to investigate how the loss of PfGlo1 or PfcGlo2 activity as well as the accumulation of AGE would affect gametocyte commitment, thus the ability of the $P$. falciparum blood stages to enter the sexual pathway and to form transmissible gametocytes. Gametocyte cultures were set up for the clonal 3D7 $\Delta$ glo1 and 3D7 $\Delta$ cglo2 parasite lines as well as the parental wild-type 3D7 line used as control. Notably, the parental 3D7 line in general was a low gametocyte producer compared to standard gametocyte-producing strains such as NF54, because 3D7 has not been selected in the past for the ability to develop gametocytes. Parasite lines 3D7 $\Delta$ glo1 and $3 \mathrm{D} 7 \Delta \mathrm{cglo} 2$ were both able to undergo gametocyte commitment and to develop mature gametocytes, and the gametocyte stages formed during maturation exhibited morphologies comparable to the wild-type strain (Fig. S1). Compared to its parental line, 3D7 $\Delta$ glo1 parasites formed reduced numbers of gametocytes in more than three independent experiments, when these were quantified 14 days after setting up the gametocyte cultures. It was noteworthy, though, that $3 D 7 \Delta \mathrm{cglo} 2$ parasites had an increased gametocytemia compared to the parental line in all of the experiments (Fig. 6), indicating that the lack of PfcGlo2 results in increased gametocyte induction.

\section{DISCUSSION}

The cytosolic $P$. falciparum glyoxalases are dispensable during asexual blood-stage development in contrast to previous analysis using pHTK vectors [13], and glyoxalase knockout parasites have no significant growth defect. Our study exemplifies that negative results from traditional 
A

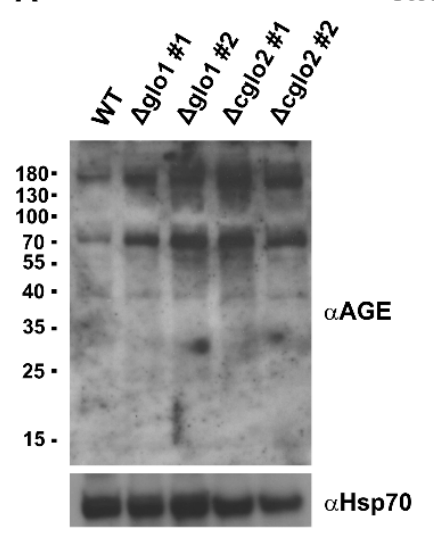

Steady state

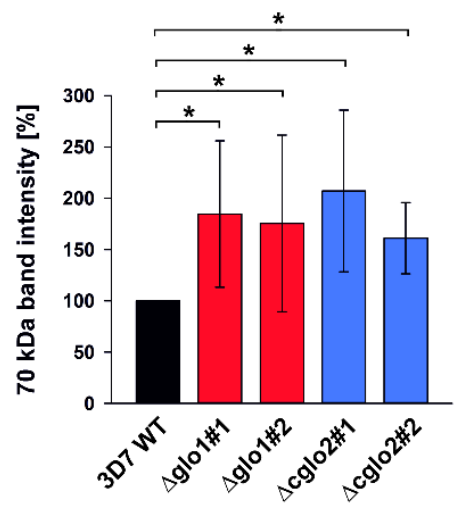

B
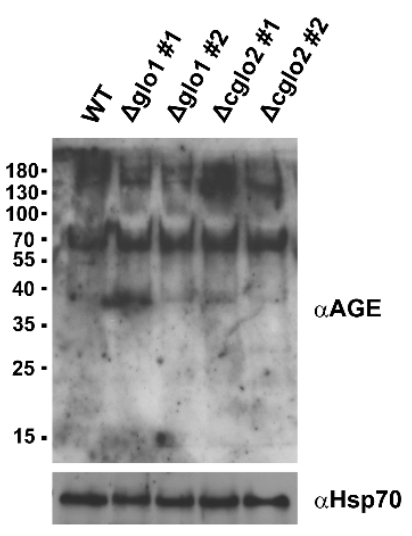

$+50 \mu \mathrm{M}$ glyoxal

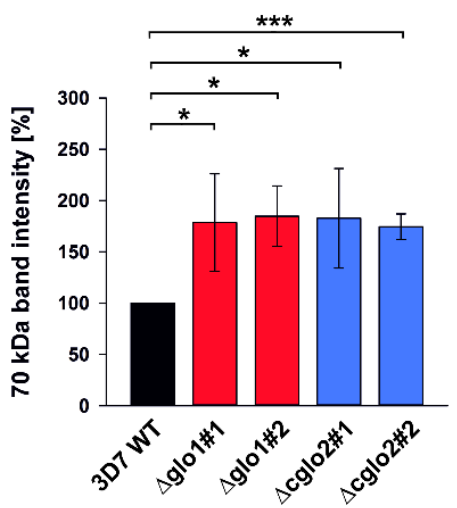

FIGURE 5: AGE detection by western blot analysis of parasite extracts from $P$. falciparum knockout strains 3D7Dglo1 and $3 D 7 \Delta$ cglo2 after erythrocyte removal by saponin lysis. Wild-type parasites served as a control. (A) AGE detection under steady-state conditions without external 2-oxoaldehydes. (B) AGE detection after a $6 \mathrm{~h}$ bolus treatment with $50 \mu \mathrm{M}$ glyoxal (which approximately corresponds to the $\mathrm{IC}_{50}$ values determined in Fig. S2). Trophozoite extracts from $2 \times 10^{7}$ cells were loaded per lane on a $15 \%$ gel, separated by reducing SDS-PAGE and decorated with a commercial polyclonal anti-AGE antibody. Decoration against Hsp70 served as a loading control on a separate blot that was run in parallel using the same parasite extracts. Signal intensities were analyzed form three independent experiments using ImageJ and statistical analyses were performed in SigmaPlot 12.5 using the one way ANOVA method $(* p<0.05 ; * * * p<0.001)$. knockout attempts in P. falciparum are difficult to interpret because of methodological limitations, even when positive and negative selections are applied [28]. Some of these limitations can be overcome by the targeted introduction of DNA double-strand breaks with the help of the CRISPRCas9 system [29]. Nevertheless, also the CRISPR-Cas9 system has limitations and four attempts of our laboratory to generate $3 D 7 \Delta$ tglo2 and $3 D 7 \Delta$ gilp knockout parasites (PlasmoDB

annotations PF3D7_1205700 and PF3D7_0604700) using pL7 plasmids have failed to date (data not shown). We consider the selection of the guide RNA or of the extremely AT-rich homology regions for PFtGLO2 and PFGILP as likely methodological causes for the negative outcome of these knockout attempts rather than essential roles of both genes. This interpretation is also supported by a recent study in the rodent parasite $P$. berghei, revealing that the homologs PBtGLO2 (PBANKA_0604400) and PBGLO1 (PBANKA_0933900) are dispensable and that knockout parasites have no growth defect [30] (the genes PBCGLO2 (PBANKA_1004000) and PBGILP (PBANKA_0103400) were not covered in the genetic screen).

The dispensability of PFGLO1 for parasite survival also explains the high $I C_{50}$ values of tight-binding Glo1-inhibitors in previous cell culture experiments [10] in contrast to low nanomolar inhibition constants against recombinant
PfGlo1 [9]. In accordance with these results, a single $\mathrm{IC}_{50}$ experiment with $3 D 7 \Delta$ glo1 and $3 D 7 \Delta c g l o 2$ knockout strains and the Glo1 inhibitors compound 3 and 7 of our previous study [10] showed very similar $I C_{50}$ values compared to wild-type parasites (data not shown). If human Glo1 compensated for the loss of PfGlo1 in the 3D7 $\Delta$ glo1 knockout strains, one would expect altered $\mathrm{IC}_{50}$ values because the inhibitors can also slowly inactivate the human enzyme [10]. Thus, parasite killing of wild-type and knockout strains at micromolar instead of nanomolar Glo1 inhibitor concentrations can be attributed to off-target effects. Regarding the dispensability of $P F C G L O 2$ for parasite survival, it might be necessary to also delete PFtGLO2 and/or to inactivate human Glo2 to cause a growth defect. However, this means that an inhibitor would have to target not only one but two or even three enzymes at once. Altogether our data suggest that PfGlo1 and PfcGlo2 are most likely not suited as targets for selective drug development.

One significant difference between wild-type and glyoxalase knockout parasites was observed for 3D7 $\Delta$ glo1 parasites that were treated with external glyoxal, whereas methylglyoxal or phenylglyoxal had similar effects on wildtype and knockout parasites. The results might be explained by different kinetics for the transport of glyoxal, methylglyoxal and phenylglyoxal across the three membranes that surround the parasite cytosol or by different 


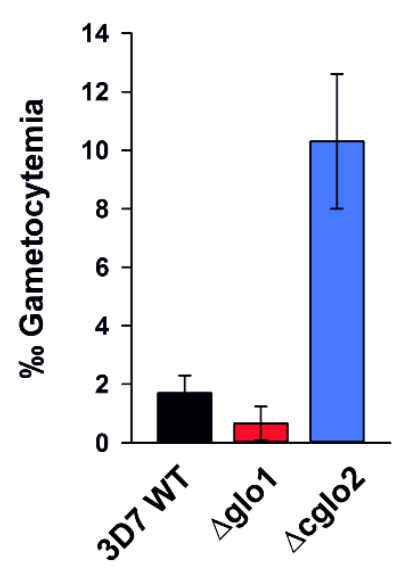

representative

independent experiments.
FIGURE 6: Gametocyte development of the $P$. falciparum knockout strains 3D7 $\Delta$ glo1 and 3D7Acglo2. Gametocyte commitment was induced in

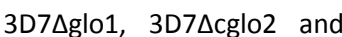
wild-type 3D7 parasites and the gametocytemia was determined per 1000 red blood cells in Giemsastained blood smears 14 days post-induction. All data points are the mean \pm S.D. from triplicate measurements. Results shown are

of

three

glyoxalase efficiencies of the host-parasite unit regarding the conversion of these 2-oxoaldehydes. A third explanation could be that other enzymes of the host-parasite unit, such as 2-oxoaldehyde reductases or dehydrogenases, are able to metabolize methylglyoxal and phenylglyoxal but not glyoxal with sufficient efficiencies in the knockout strains.

An interesting observation was that the loss of PfcGlo2 in the asexual blood stages resulted in increased gametocyte formation. Commitment to gametocyte development is believed to take place at some point before schizogony, when individual schizonts produce merozoites that develop into gametocytes after invasion of red blood cells [31, 32]. A number of recent studies have demonstrated that the switch between asexual blood stage replication and gametocyte formation in $P$. falciparum is epigenetically regulated, involving $P$. falciparum heterochromatin protein 1 and histone deacetylase 2, as well as down-stream transcription factors such as Ap2G [33-36]. Gametocyte commitment is assigned to a variety of internal and external stress factors such as high parasitemia, anemia, host immune response or drug treatment, but also endoplasmic reticulum stress [36-43]. AGE accumulation appeared to be similar in 3D7 $\Delta$ glo1 and 3D7 $\Delta$ cglo2 knockout parasites and the lack of functional PfGlo1 did not trigger gametocyte commitment. We therefore speculate that the accumulation of $S$-D-lactoylglutathione or another thioester substrate of PfcGlo2 $[1,3,8]$ might have caused the induction of gametocyte commitment. Altered S-D-lactoylglutathione concentrations were previously reported to activate Escherichia coli potassium channels that share properties with cation-translocating channels in eukaryotes [20, 24, 44]. Furthermore, altered S-D-lactoylglutathione concentrations might also affect the uptake of glutathione into organelles as previously reported for isolated rat liver mitochondria [25]. Whether similar S-D-lactoylglutathionedependent mechanisms play a role in gametocyte commitment remains to be studied.

\section{MATERIALS AND METHODS \\ Materials}

The Cas9-encoding plasmid pUF1-Cas9 and plasmid pL6, containing the single guide RNA (sgRNA) with a Cas9-binding sequence, were generously provided by Jose-Juan LopezRubio [29]. Cloning vector PCRII-TOPO was from Life technologies. Primers were from Metabion and Taq DNA polymerase and restriction enzymes were from New England Biolabs. The In-Fusion HD cloning kit was from Clontech. RPMI-1640 medium and albumax II were from Gibco Life Technologies. Hypoxanthine and gentamicin were from C.C.Pro $\mathrm{GmbH}$ or Gibco Life Technologies. Peptides and rabbit peptide antibodies against PfGlo1 ( $\mathrm{NH}_{2}$-CLKYQTDEDYENFKQSWEPV-CONH $\left.{ }_{2}\right)$ and PfCGlo2 $\left(\mathrm{NH}_{2}\right.$-CSAYEPTPGVNEKVYDGQ-CONH$\left.{ }_{2}\right)$ were generated by Pineda Antibody Service. SulfoLink resin was from Pierce. The polyclonal anti-AGE antibody (ab23722) was from Abcam and the goat anti-Rabbit IgG $(\mathrm{H}+\mathrm{L})$-HRP conjugate was from BioRad. Saponin, blotting-grade milk powder, glyoxal, methylglyoxal and phenylglyoxal were from Sigma.

\section{Cloning of pL7 constructs}

The pL7 transfection plasmids containing the single guide RNA (sgRNA) expression-cassette and the 5'- and 3'-homology regions of PFGLO1 and PFCGLO2 (PlasmoDB annotations PF3D7_1113700 and PF3D7_0406400) were generated from plasmid pL6 by multiple cloning steps. First, to generate plasmid pL6-PFGLO1, the $5^{\prime}$ - and $3^{\prime}$-homology regions of PFGLO1 were PCR-amplified from $P$. falciparum strain 3D7 genomic DNA using primer sets $P 1 / P 2$ and $P 3 / P 4$, respectively (Table S1). PCR products were initially cloned into pCRII-TOPO. The $5^{\prime}$ - and $3^{\prime}$-homology regions were subsequently excised from the TOPO vector, using EcoRI/Ncol for the $3^{\prime}$-fragment and Sacll/Xbal for the 5'-fragment, and cloned into pL6, using the restriction sites EcoRI/Ncol for the 3'-fragment and Sacll/Spel for the $5^{\prime}$-fragment. Plasmid pL6-PFCGLO2 was cloned analogously using primers set P5/P6 and P7/P8 (Table S1). Plasmids pL7-PFGLO1 and pL7-PFCGLO2 were subsequently generated by replacing the BtgZl-adaptor of pL6-PFGLO1 and pL6PFCGLO2 with the guide RNA (gRNA) fragment using the InFusion HD cloning kit. Briefly, the pL6 plasmids were digested stepwise with Avrll and BtgZl for $2 \mathrm{~h}$ at $37^{\circ} \mathrm{C}$ and $2 \mathrm{~h}$ at $60^{\circ} \mathrm{C}$, respectively. The 20-base gRNA fragments for each target were designed using the Protospacer software, flanked with 15 bases for In-Fusion cloning and purchased as complementary oligonucleotides $\mathrm{P} 9 / \mathrm{P} 10$ and $\mathrm{P} 11 / \mathrm{P} 12$ for PL7-PFGLO1 and pL7-PFCGLO2, respectively (Table S1). The complementary oligonucleotides were mixed, annealed and ligated with the purified linearized pL6 plasmids in the In-fusion reaction. Stellar chemical competent cells were subsequently transformed with the In-Fusion reaction products. Correct sequences for the $5^{\prime}$ - and $3^{\prime}$-homology regions as well as the sgRNA cassettes of pL7-PFGLO1 and pL7-PFCGLO2 were confirmed by commercial DNA sequencing at GATC Biotech.

\section{Parasite culture and transfections}

Asexual $P$. falciparum blood-stage parasites were cultured in fresh human A+ erythrocytes according to Trager and Jensen [45] at $36.5^{\circ} \mathrm{C}, 5 \% \mathrm{O}_{2}, 5 \% \mathrm{CO}_{2}$ and $80 \%$ humidity in RPMI-1640 medium supplemented with $0.45 \%$ albumax II, $0.2 \mathrm{mM}$ hypoxanthine and $2.7 \mu \mathrm{g} / \mathrm{ml}$ gentamicin. The hematocrit was $3.5 \%$ and the standard culture volume was $14 \mathrm{ml}$. Synchronised parasite cultures were obtained after treatment with 
5\% sorbitol [46]. Transfections were conducted by electroporation (pre-loading) of uninfected erythrocytes with $100 \mu \mathrm{g}$ pUF1-Cas9 or pL7 at $0.3 \mathrm{kV}$ and $950 \mu \mathrm{F}$ followed by the infection with $P$. falciparum 3D7 parasites $[47,48]$. Parasites were first transfected with plasmid pUF1-Cas9 and selected with $100 \mathrm{nM}$ atovaquone followed by transfection with plasmid pL7-PFGLO1 or pL7-PFCGLO2 and selection with $2.7 \mathrm{nM}$ WR99210. The drug pressure was applied $20 \mathrm{~h}$ posttransfection. For the first 7 days, drug and medium were renewed every $24 \mathrm{~h}$. Thereafter, drug and medium were renewed every other day, and $70 \mu \mathrm{l}$ of fresh erythrocytes were added to the culture once every week until parasites were detected in Giemsa-stained blood smears. A negative selection against parasites with non-integrated pL7 plasmids was conducted with $40 \mu \mathrm{M}$ 5-flourocytosine. Clonal cell lines were obtained by limited dilution in 96 -well plates.

\section{PCR analysis of parasite strains}

The uptake of pUF1-Cas9 and the targeted disruption of the glyoxalases by the insertion of the dihydrofolate reductase gene were verified by PCR analysis using primer pairs P13/P14 for pUF1-Cas9, P15/P16, P15/P17, and P18/P16 for the disruption of PFGLO1 and P19/P20, P19/P17, and P18/P20 for the disruption of PFCGLO2 (Table S1). Genomic DNA for PCR analysis was isolated with phenol/chloroform from late schizont/early ring-stage parasites at approximately $8 \%$ parasitemia as described previously [49].

\section{Purification of peptide antibodies}

Rabbit peptide antibodies against PfGlo1 and PfcGlo2 were purified from sera of immunized animals by affinity chromatography. In brief, cysteine-containing peptides $(1 \mathrm{mg} / \mathrm{ml})$ were reduced with $25 \mathrm{mM}$ borohydride in $50 \mathrm{mM}$ Tris/ $\mathrm{HCl}, 5 \mathrm{mM}$ EDTA, pH 8.5 at $4^{\circ} \mathrm{C}$ overnight. The reduced peptides were coupled for $45 \mathrm{~min}$ to $1 \mathrm{ml}$ SulfoLink resin. Unspecific binding sites were subsequently blocked with $50 \mathrm{mM}$ cysteine in 50 $\mathrm{mM}$ Tris, $5 \mathrm{mM}$ EDTA, pH 8.5. Peptide antibodies were affinitypurified from rabbit sera according to the manufacturer's protocol. Antibody-containing eluate fractions were identified after SDS-polyacrylamide gel electrophoresis (SDS-PAGE) on Coomassie-stained $15 \%$ gels.

\section{Western blot analysis}

Trophozoite extracts were prepared by saponin lysis [50, 51] from $35 \mathrm{ml}$ cultures with $5 \%$ parasitemia. Briefly, after centrifugation at room temperature for 5 minutes at $755 \mathrm{~g}$, erythrocytes were resuspended in ice-cold phosphate buffered saline (PBS) containing $0.05 \%(w / v)$ saponin and incubated for $60 \mathrm{sec}$ on ice. The erythrocyte lysate was centrifuged at $4^{\circ} \mathrm{C}$ for $10 \mathrm{~min}$ at $1800 \mathrm{~g}$. The supernatant was discarded and the parasite pellet was washed twice with PBS and once with PBS containing Roche complete protease inhibitor (PBS/PI). Purified parasites were resuspended in $50 \mu \mathrm{l}$ of PBS/PI and disrupted by four freeze-thaw cycles comprising 5 min freezing in liquid nitrogen, $1 \mathrm{~min}$ thawing in a $37^{\circ} \mathrm{C}$ water bath and 30 sec of mixing on a vortex. The parasite extract was supplemented with $50 \mu \mathrm{l}$ of $2 \times$ Laemmli buffer containing $30 \%(\mathrm{v} / \mathrm{v})$ 2-mercapto-ethanol and boiled at $95^{\circ} \mathrm{C}$ for $5 \mathrm{~min}$. Protein extracts of approximately $2 \times 10^{7}$ purified trophozoites per lane were separated by SDS-PAGE and subsequently transferred to a nitrocellulose membrane by semi-dry blotting. The membrane was blocked for $1 \mathrm{~h}$ at room temperature with $5 \%(\mathrm{w} / \mathrm{v})$ milk powder in $0.9 \%(\mathrm{w} / \mathrm{v}) \mathrm{NaCl}, 10 \mathrm{mM}$ Tris/HCl, $\mathrm{pH} 7.4$ (milk/TBS) and incubated with preimmune serum, serum or purified antibodies in milk/TBS at $4^{\circ} \mathrm{C}$ overnight. For the detection of PfGlo1, PfcGlo2 and AGE, the purified peptide antibodies $\alpha$ PfGlo1 and $\alpha$ PfcGlo2 as well as the commercial anti-AGE antibody were diluted 1:500, 1:1000 and 1:500, respectively. The secondary goat anti-Rabbit antibody was diluted 1:10000 in $5 \%$ milk/TBS. At least three biological replicates were performed for each western blot analysis. Signal intensities were analyzed with ImageJ [52].

\section{Growth curve analysis}

The growth phenotype of three clonal 3D7 $\Delta$ glo1 and two clonal $3 D 7 \Delta$ cglo2 knockout strains was analysed in comparison with the 3D7 wild-type strain. Asynchronous standard cultures of each strain were adjusted to a starting parasitemia of $0.1 \%$ and maintained for six days. The medium was changed once every $24 \mathrm{~h}$ for the first three days and then twice every $24 \mathrm{~h}$. Parasite morphology and parasitemia of each culture were daily assessed by light microscopy. Approximately $750-1500$ erythrocytes were analyzed per Giemsa-stained blood smear. The average parasitemia was calculated for each time point from three independent biological replicates. Statistical analysis was performed in SigmaPlot 12.5 using the one way ANOVA method. Growth inhibition of synchronous ring-stage

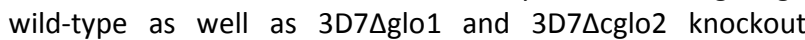
parasites by exogenous glyoxal, methylglyoxal or phenylglyoxal was determined in 96-well plates using a SYBR green 1 assay as described previously $[53,54]$. Briefly, $72 \mathrm{~h}$ after the bolus treatments, the SYBR green fluorescence was measured in a microplate reader (BMG Latech, Germany), and $I_{50}$ values were computed from sigmoidal dose-response curves using the four parameter Hill function in SigmaPlot 12.5.

\section{Gametocyte formation assay}

Wild-type as well as $3 D 7 \Delta$ glo1 and $3 D 7 \Delta$ cglo2 knockout parasites were cultured as described above except for the replacement of albumax with $10 \%$ inactivated $A+$ serum as described previously [55]. Two days after synchronization with $5 \%$ sorbitol, gametocyte commitment was induced by setting up the culture at $2 \%$ parasitemia and a haematocrit of $10 \%$ in $5 \mathrm{ml}$ of the human A+ medium. The medium was replaced daily and Giemsa-stained blood smears were analyzed $14 \mathrm{~d}$ post-induction of gametocyte commitment. The gametocytemia was determined per 1000 red blood cells and the gametocyte stages II-V were counted in triplicate. Data analysis was performed using MS Excel 2010 and GraphPad Prism 5.

\section{Glyoxalase activity assays}

Parasite lysates for glyoxalase activity measurements were prepared from $35 \mathrm{ml}$ cultures of synchronous trophozoitestage parasites using a slightly modified lysis protocol [7]. Briefly, infected erythrocytes were first pelleted and then resuspended and lysed in 20 volumes of modified PBS containing $7 \mathrm{mM} \mathrm{K}_{2} \mathrm{HPO}_{4}, 1 \mathrm{mM} \mathrm{NaH}_{2} \mathrm{PO}_{4}, 11 \mathrm{mM} \mathrm{NaHCO}, 58 \mathrm{mM} \mathrm{KCl}$, $56 \mathrm{mM} \mathrm{NaCl}, 1 \mathrm{mM} \mathrm{MgCl} 2,14 \mathrm{mM}$ glucose and $0.02 \%(\mathrm{w} / \mathrm{v})$ saponin $\left(\mathrm{pH} 7.5\right.$ at $25^{\circ} \mathrm{C}$ ). After incubation for $10 \mathrm{~min}$ at $37^{\circ} \mathrm{C}$, erythrocyte lysates were centrifuged at $25^{\circ} \mathrm{C}$ for $3 \mathrm{~min}$ at 1500 $g$ and the pellet was washed three times with modified PBS. About $5 \times 10^{7}$ purified trophozoites were resuspended in 150 $\mu \mathrm{l}$ of modified PBS and disrupted by four freeze-thaw cycles. The parasite lysate was centrifuged at $4^{\circ} \mathrm{C}$ for $60 \mathrm{~min}$ at 20800 
$g$ and the supernatant was transferred to a fresh precooled reaction tube and stored on ice for immediate Glo1 and Glo2 activity assays. The protein content of the parasite lysate supernatant was subsequently determined in a Bradford assay at $595 \mathrm{~nm}$ with bovine serum albumin as a standard [56].

Glo1 activities form parasite extracts were determined at $25^{\circ} \mathrm{C}$ using a thermostatted Jasco V-550 UV-vis spectrophotometer by following the formation of $S$-D-lactoylglutathione and the consumption of the hemithioacetal between methylglyoxal and reduced glutathione (GSH) at $240 \mathrm{~nm}$ using an extinction coefficient of $\varepsilon_{240 \mathrm{~nm}}=2.86 \mathrm{mM}^{-1} \mathrm{~cm}^{-1}[10,57]$. Briefly, stock solutions of $100 \mathrm{mM} \mathrm{GSH}$ and $100 \mathrm{mM}$ methylglyoxal were freshly prepared in water and stored on ice. The hemithioacetal substrate was formed by mixing $100 \mu \mathrm{l}$ of prewarmed $1 \mathrm{M} \mathrm{K}_{\mathrm{x}} \mathrm{H}_{\mathrm{y}} \mathrm{PO}_{4}, \mathrm{pH} 7.0$ and $100 \mu \mathrm{l} 1 \mathrm{M} \mathrm{KCl}$ with $10 \mu \mathrm{l}$ of $100 \mathrm{mM} \mathrm{GSH}$ and $20 \mu \mathrm{l}$ of $100 \mathrm{mM}$ methylglyoxal in $740 \mu \mathrm{l}$ of pre-warmed double distilled water in a $1 \mathrm{ml}$ cuvette. The mixture was incubated for $15 \mathrm{~min}$ at $25^{\circ} \mathrm{C}$. After $14 \mathrm{~min}$ incubation, the measurement was set to 'auto zero', and a baseline was recorded after $14.5 \mathrm{~min}$ for $30 \mathrm{sec}$ before adding $30 \mu \mathrm{l}$ of parasite lysate supernatant from approximately $10^{7}$ trophozoites to the mixture. After thorough mixing, the change in absorbance at $A_{240}$ was immediately monitored. The slope of the initial increase of absorbance over time $\left(\mathrm{d} A_{240} / \mathrm{d} t\right)_{0}$ was determined and the activity of Glo1 $\left(\mathrm{a}_{\mathrm{Glo1}}\right)$ was calculated using $\mathrm{a}_{\mathrm{Glo} 1}=\left(\mathrm{d} A_{240} / \mathrm{d} t\right)_{0} / 2.86[\mathrm{mM} / \mathrm{min}]$.

Glo2 activities were measured analogously at $25^{\circ} \mathrm{C}$ by following the consumption of S-D-lactoylglutathione at $240 \mathrm{~nm}$ using an extinction coefficient of $\varepsilon_{240 \mathrm{~nm}}=3.1 \mathrm{mM}^{-1} \mathrm{~cm}^{-1}[10$, 57]. Briefly, $100 \mathrm{mM} \mathrm{Tris} / \mathrm{HCl}, \mathrm{pH} 7.4$ at $25^{\circ} \mathrm{C}$ and $3 \mathrm{mM}$ $S$-D-lactoylglutathione in Tris buffer were freshly prepared and stored on ice. The assay setup comprised mixing $500 \mu \mathrm{l}$ of pre-warmed Tris buffer, $100 \mu \mathrm{l}$ of $3 \mathrm{mM} \mathrm{S}$-D-lactoylglutathione and $350 \mu \mathrm{l}$ of pre-warmed water in a $1 \mathrm{ml}$ cuvette at $25^{\circ} \mathrm{C}$. The measurement was set to 'auto zero' and a baseline was recorded for 30 seconds before adding $50 \mu$ l of parasite lysate supernatant from approximately $1.7 \times 10^{7}$ trophozoites to the mixture. After thorough mixing, the change in absorbance at $A_{240}$ was immediately monitored. The slope of the initial decrease of absorbance over time $\left(\mathrm{d} A_{240} / \mathrm{d} t\right)_{0}$ was determined and the activity of $\mathrm{Glo} 2\left(\mathrm{a}_{\mathrm{Glo} 2}\right)$ was calculated using $\mathrm{a}_{\mathrm{Glo} 2}=-\left(\mathrm{d} A_{240} / \mathrm{d} t\right)_{0} / 3.1[\mathrm{mM} / \mathrm{min}]$.

\section{REFERENCES}

1. Vander Jagt DL, Hunsaker LA, Campos NM, Baack BR (1990). Dlactate production in erythrocytes infected with Plasmodium falciparum. Mol Biochem Parasitol 42(2): 277-284. PMID: 2270109

2. Deponte M, Sturm N, Mittler S, Harner M, Mack H, Becker K (2007). Allosteric coupling of two different functional active sites in monomeric Plasmodium falciparum glyoxalase I. J Biol Chem 282(39): 28419-28430. doi: 10.1074/jbc.M703271200

3. Urscher M, Deponte M (2009). Plasmodium falciparum glyoxalase II: Theorell-Chance product inhibition patterns, rate-limiting substrate binding via $\operatorname{Arg}(257) / \operatorname{Lys}(260)$, and unmasking of acid-base catalysis. Biol Chem 390(11): 1171-1183. doi: 10.1515/BC.2009.127

4. Urscher M, Przyborski JM, Imoto M, Deponte M (2010). Distinct subcellular localization in the cytosol and apicoplast, unexpected dimerization and inhibition of Plasmodium falciparum glyoxalases. Mol Microbiol 76(1): 92-103. doi: 10.1111/j.1365-2958.2010.07082.x
Glo1 and Glo2 activities were corrected for the dilution factor of the parasite lysate and normalized to the protein content of the lysate (yielding $\mathrm{mU} / \mathrm{mg}$ (total protein) with $1 \mathrm{mU}$ being the amount of enzyme that converts $1 \mathrm{nmol}$ of substrate per $\mathrm{min}$ ). The normalized Glo1 and Glo2 activities were averaged from two to four independent triplicate measurements. A statistical analysis was performed in SigmaPlot 12.5 using the one way ANOVA method.

\section{ACKNOWLEDGMENTS}

The position of C.A.W. was funded by the German Academic Exchange Service (DAAD) and the Ministry of Education, Ghana. L.L. and V.S. were funded by the DFG (grants DE 1431/8-1 and DE 1431/10-1 to M.D.). M.D. and G.P. are grateful to the DFG for funding of their positions in the frame of the Heisenberg program (grants DE 1431/9-1 and PR905/8-1 and 8-2). We thank Jose-Juan Lopez-Rubio for plasmids pUF1-Cas9 and pL6.

\section{SUPPLEMENTAL MATERIAL}

All supplemental data for this article are available online at www.microbialcell.com.

\section{CONFLICT OF INTEREST}

The authors declare no conflict of interest.

\section{COPYRIGHT}

(C) 2017 Wezena et al. This is an open-access article released under the terms of the Creative Commons Attribution (CC BY) license, which allows the unrestricted use, distribution, and reproduction in any medium, provided the original author and source are acknowledged.

Please cite this article as: Cletus A. Wezena, Romy Alisch, Alexandra Golzmann, Linda Liedgens, Verena Staudacher, Gabriele Pradel and Marcel Deponte (2017). The cytosolic glyoxalases of Plasmodium falciparum are dispensable during asexual bloodstage development. Microbial Cell 5(1): 32-41. doi: 10.15698/mic2018.01.609

5. Deponte M (2013). Glutathione catalysis and the reaction mechanisms of glutathione-dependent enzymes. Biochim Biophys Acta 1830(5): 3217-3266. doi: 10.1016/j.bbagen.2012.09.018.

6. Thornalley PJ, Strath M, Wilson RJ (1994). Antimalarial activity in vitro of the glyoxalase I inhibitor diester, S-p-bromobenzylglutathione diethyl ester. Biochem Pharmacol 47(2): 418-420. PMID: 8304987

7. Akoachere $M$, lozef R, Rahlfs S, Deponte $M$, Mannervik B, Creighton DJ, Schirmer H, Becker K (2005). Characterization of the glyoxalases of the malarial parasite Plasmodium falciparum and comparison with their human counterparts. Biol Chem 386(1): 41-52. doi: 10.1515/BC.2005.006

8. Urscher M, Alisch R, Deponte M (2011). The glyoxalase system of malaria parasites--implications for cell biology and general glyoxalase research. Semin Cell Dev Biol 22(3): 262-270. doi: 10.1016/j.semcdb.2011.02.003 
9. Urscher M, More SS, Alisch R, Vince R, Deponte M (2012). Tightbinding inhibitors efficiently inactivate both reaction centers of monomeric Plasmodium falciparum glyoxalase 1. FEBS J 279(14): 25682578. doi: 10.1111/j.1742-4658.2012.08640.x

10. Wezena CA, Urscher M, Vince R, More SS, Deponte M (2016). Hemolytic and antimalarial effects of tight-binding glyoxalase 1 inhibitors on the host-parasite unit of erythrocytes infected with Plasmodium falciparum. Redox Biol 8:348-353. doi: 10.1016/j.redox.2016.02.006

11. Suttisansanee U, Honek JF (2011). Bacterial glyoxalase enzymes. Semin Cell Dev Biol 22(3): 285-292. doi: 10.1016/j.semcdb.2011.02.004

12. Sousa Silva $M$, Gomes RA, Ferreira AE, Ponces Freire A, Cordeiro C (2013). The glyoxalase pathway: the first hundred years and beyond. Biochem J 453(1): 1-15. doi: 10.1042/BJ20121743

13. Deponte M (2014). Glyoxalase diversity in parasitic protists. Biochem Soc Trans 42(2): 473-478. doi: 10.1042/BST20140005

14. Vince R, Wadd WB (1969). Glyoxalase inhibitors as potential anticancer agents. Biochem Biophys Res Commun 35(5): 593-598. PMID: 5794079

15. Thornalley PJ, Rabbani N (2011). Glyoxalase in tumourigenesis and multidrug resistance. Semin Cell Dev Biol 22(3): 318-325. doi: 10.1016/j.semcdb.2011.02.006

16. Brouwers O, Niessen PM, Ferreira I, Miyata T, Scheffer PG, Teerlink T, Schrauwen P, Brownlee M, Stehouwer CD, Schalkwijk CG (2011). Overexpression of glyoxalase-I reduces hyperglycemia-induced levels of advanced glycation end products and oxidative stress in diabetic rats. J Biol Chem 286(2): 1374-1380. doi: 10.1074/jbc.M110.144097

17. Rabbani N, Thornalley PJ (2011). Glyoxalase in diabetes, obesity and related disorders. Semin Cell Dev Biol 22(3): 309-317. doi: 10.1016/j.semcdb.2011.02.015

18. Fleming T, Nawroth PP (2014). Reactive metabolites as a cause of late diabetic complications. Biochem Soc Trans 42(2): 439-442. doi: 10.1042/BST20130265

19. Brings $S$, Fleming $T$, Freichel $M$, Muckenthaler MU, Herzig $S$, Nawroth PP (2017). Dicarbonyls and Advanced Glycation End-Products in the Development of Diabetic Complications and Targets for Intervention. Int J Mol Sci 18(5). doi: 10.3390/ijms18050984

20. Ferguson GP, Munro AW, Douglas RM, McLaggan D, Booth IR (1993). Activation of potassium channels during metabolite detoxification in Escherichia coli. Mol Microbiol 9(6): 1297-1303. PMID: 7934942

21. Maeta K, Izawa S, Okazaki S, Kuge S, Inoue Y (2004). Activity of the Yap1 transcription factor in Saccharomyces cerevisiae is modulated by methylglyoxal, a metabolite derived from glycolysis. Mol Cell Biol 24(19): 8753-8764. doi: 10.1128/MCB.24.19.8753-8764.2004

22. Kawatani M, Okumura $\mathrm{H}$, Honda K, Kanoh $\mathrm{N}$, Muroi M, Dohmae N, Takami M, Kitagawa M, Futamura Y, Imoto M, Osada H (2008). The identification of an osteoclastogenesis inhibitor through the inhibition of glyoxalase I. Proc Natl Acad Sci U S A 105(33): 11691-11696. doi: 10.1073/pnas.0712239105

23. Wendler A, Irsch T, Rabbani N, Thornalley PJ, Krauth-Siegel RL (2009). Glyoxalase II does not support methylglyoxal detoxification but serves as a general trypanothione thioesterase in African trypanosomes. Mol Biochem Parasitol 163(1): 19-27. doi: 10.1016/j.molbiopara.2008.09.005

24. Ozyamak E, Black SS, Walker CA, Maclean MJ, Bartlett W, Miller S, Booth IR (2010). The critical role of S-lactoylglutathione formation during methylglyoxal detoxification in Escherichia coli. Mol Microbiol 78(6): 1577-1590. doi: 10.1111/j.1365-2958.2010.07426.x
25. Armeni T, Cianfruglia L, Piva F, Urbanelli L, Luisa Caniglia M, Pugnaloni A, Principato $G$ (2014). S-D-Lactoylglutathione can be an alternative supply of mitochondrial glutathione. Free Radic Biol Med 67:451459. doi: 10.1016/j.freeradbiomed.2013.12.005

26. Schimandle CM, Vander Jagt DL (1979). Isolation and kinetic analysis of the multiple forms of glyoxalase-I from human erythrocytes. Arch Biochem Biophys 195(2): 261-268. PMID: 475391

27. Allen RE, Lo TW, Thornalley PJ (1993). Purification and characterisation of glyoxalase II from human red blood cells. Eur J Biochem 213(3): 1261-1267. PMID: 8504817

28. Duraisingh MT, Triglia T, Cowman AF (2002). Negative selection of Plasmodium falciparum reveals targeted gene deletion by double crossover recombination. Int J Parasitol 32(1): 81-89. PMID: 11796125

29. Ghorbal M, Gorman M, Macpherson CR, Martins RM, Scherf A, Lopez-Rubio JJ (2014). Genome editing in the human malaria parasite Plasmodium falciparum using the CRISPR-Cas9 system. Nat Biotechnol 32(8): 819-821. doi: $10.1038 /$ nbt.2925

30. Bushell E, Gomes AR, Sanderson T, Anar B, Girling G, Herd C, Metcalf T, Modrzynska K, Schwach F, Martin RE, Mather MW, McFadden GI, Parts L, Rutledge GG, Vaidya AB, Wengelnik K, Rayner JC, Billker $O$ (2017). Functional Profiling of a Plasmodium Genome Reveals an Abundance of Essential Genes. Cell 170(2): 260-272 e268. doi: 10.1016/j.cell.2017.06.030

31. Bruce MC, Alano P, Duthie S, Carter R (1990). Commitment of the malaria parasite Plasmodium falciparum to sexual and asexual development. Parasitology 100 (Pt 2):191-200. PMID: 2189114

32. Smith TG, Lourenco P, Carter R, Walliker D, Ranford-Cartwright LC (2000). Commitment to sexual differentiation in the human malaria parasite, Plasmodium falciparum. Parasitology 121 (Pt 2):127-133. PMID: 11085232

33. Kafsack BF, Rovira-Graells N, Clark TG, Bancells C, Crowley VM, Campino SG, Williams AE, Drought LG, Kwiatkowski DP, Baker DA, Cortes A, Llinas M (2014). A transcriptional switch underlies commitment to sexual development in malaria parasites. Nature 507(7491): 248-252. doi: 10.1038/nature12920

34. Sinha A, Hughes KR, Modrzynska KK, Otto TD, Pfander C, Dickens NJ, Religa AA, Bushell E, Graham AL, Cameron R, Kafsack BFC, Williams $A E$, Llinas $M$, Berriman $M$, Billker $O$, Waters AP (2014). A cascade of DNA-binding proteins for sexual commitment and development in Plasmodium. Nature 507(7491): 253-257. doi: 10.1038/nature12970

35. Coleman BI, Skillman KM, Jiang RHY, Childs LM, Altenhofen LM Ganter M, Leung Y, Goldowitz I, Kafsack BFC, Marti M, Llinas M, Buckee CO, Duraisingh MT (2014). A Plasmodium falciparum histone deacetylase regulates antigenic variation and gametocyte conversion. Cell Host Microbe 16(2): 177-186. doi: 10.1016/j.chom.2014.06.014

36. Brancucci NM, Bertschi NL, Zhu L, Niederwieser I, Chin WH, Wampfler R, Freymond C, Rottmann M, Felger I, Bozdech Z, Voss TS (2014). Heterochromatin protein 1 secures survival and transmission of malaria parasites. Cell Host Microbe 16(2): 165-176. doi: 10.1016/j.chom.2014.07.004

37. Smalley ME, Brown J (1981). Plasmodium falciparum gametocytogenesis stimulated by lymphocytes and serum from infected Gambian children. Trans R Soc Trop Med Hyg 75(2): 316-317. PMID: 7029805

38. Schneweis S, Maier WA, Seitz HM (1991). Haemolysis of infected erythrocytes--a trigger for formation of Plasmodium falciparum gametocytes? Parasitol Res 77(5): 458-460. PMID: 1891456

39. Trager W, Gill GS (1992). Enhanced gametocyte formation in young erythrocytes by Plasmodium falciparum in vitro. J Protozool 39(3): 429-432. PMID: 1640389 
40. Lingnau A, Margos G, Maier WA, Seitz HM (1993). The effects of hormones on the gametocytogenesis of Plasmodium falciparum in vitro. Appl Parasitol 34(3): 153-160. PMID: 8220571

41. Gautret P, Miltgen F, Gantier JC, Chabaud AG, Landau I (1996). Enhanced gametocyte formation by Plasmodium chabaudi in immature erythrocytes: pattern of production, sequestration, and infectivity to mosquitoes. J Parasitol 82(6): 900-906. PMID: 8973397

42. Puta C, Manyando C (1997). Enhanced gametocyte production in Fansidar-treated Plasmodium falciparum malaria patients: implications for malaria transmission control programmes. Trop Med Int Health 2(3): 227-229. PMID: 9491100

43. Talman AM, Paul RE, Sokhna CS, Domarle O, Ariey F, Trape JF, Robert V (2004). Influence of chemotherapy on the Plasmodium gametocyte sex ratio of mice and humans. Am J Trop Med Hyg 71(6): 739-744. PMID: 15642963

44. Roosild TP, Castronovo S, Healy J, Miller S, Pliotas C, Rasmussen T, Bartlett W, Conway SJ, Booth IR (2010). Mechanism of ligand-gated potassium efflux in bacterial pathogens. Proc Natl Acad Sci U S A 107(46): 19784-19789. doi: 10.1073/pnas.1012716107

45. Trager W, Jensen JB (1976). Human malaria parasites in continuous culture. Science 193(4254): 673-675. PMID: 781840

46. Lambros C, Vanderberg JP (1979). Synchronization of Plasmodium falciparum erythrocytic stages in culture. J Parasitol 65(3): 418-420. PMID: 383936

47. Deitsch K, Driskill C, Wellems T (2001). Transformation of malaria parasites by the spontaneous uptake and expression of DNA from human erythrocytes. Nucleic Acids Res 29(3): 850-853. PMID: 11160909

48. Hasenkamp S, Russell KT, Horrocks P (2012). Comparison of the absolute and relative efficiencies of electroporation-based transfection protocols for Plasmodium falciparum. Malar J 11:210. doi: 10.1186/1475-2875-11-210

49. Beck HP (2002). Extraction and purification of Plasmodium parasite DNA. Methods Mol Med 72:159-163. doi: 10.1385/1-59259-271$6: 159$
50. Benting J, Mattei D, Lingelbach K (1994). Brefeldin A inhibits transport of the glycophorin-binding protein from Plasmodium falciparum into the host erythrocyte. Biochem J 300 (Pt 3):821-826. PMID: 8010965

51. Zuckerman A, Spira D, Hamburger J (1967). A procedure for the harvesting of mammalian plasmodia. Bull World Health Organ 37(3): 431-436. PMID: 4968349

52. Schneider CA, Rasband WS, Eliceiri KW (2012). NIH Image to ImageJ: 25 years of image analysis. Nat Methods 9(7): 671-675. PMID: 22930834

53. Wezena CA, Krafczyk J, Staudacher V, Deponte M (2017). Growth inhibitory effects of standard pro- and antioxidants on the human malaria parasite Plasmodium falciparum. Exp Parasitol 180:64-70. doi: 10.1016/j.exppara.2017.02.017

54. Smilkstein $M$, Sriwilaijaroen $N$, Kelly JX, Wilairat $P$, Riscoe $M$ (2004). Simple and inexpensive fluorescence-based technique for high-throughput antimalarial drug screening. Antimicrob Agents Chemother 48(5): 1803-1806. PMID: 15105138

55. Wirth CC, Glushakova S, Scheuermayer M, Repnik U, Garg S, Schaack D, Kachman MM, Weissbach T, Zimmerberg J, Dandekar T, Griffiths G, Chitnis CE, Singh S, Fischer R, Pradel G (2014). Perforin-like protein PPLP2 permeabilizes the red blood cell membrane during egress of Plasmodium falciparum gametocytes. Cell Microbiol 16(5): 709-733. doi: 10.1111/cmi.12288

56. Bradford MM (1976). A rapid and sensitive method for the quantitation of microgram quantities of protein utilizing the principle of protein-dye binding. Anal Biochem 72:248-254. PMID: 942051

57. Arai M, Nihonmatsu-Kikuchi N, Itokawa M, Rabbani N, Thornalley PJ (2014). Measurement of glyoxalase activities. Biochem Soc Trans 42(2): 491-494. doi: 10.1042/BST20140010 